\title{
Optimizing \& Automating EELS/EFTEM Parameters Using Spectrum Simulation
}

\author{
John A. Hunt \& Nanda K. Menon \\ Gatan Research \& Development, 5933 Coronado Lane, Pleasanton, CA 94588 USA
}

Electron energy-loss spectroscopy (EELS) in the TEM is widely recognized as a microanalytical tool capable of characterizing the elemental composition of materials with a spatial resolution approaching atomic scale and sensitivity approaching the single atom level. Due to the richness of the information in the EELS signal and complexity of the background shape, extracting quantitative compositional information from EELS data can be highly subjective and dependent on user experience. Despite these difficulties, EELS is often the analysis method of choice due to its high detection efficiency which often translates to improved sensitivity. ${ }^{1}$ This is often the case when dose is limited by the necessity to work with STEM probes at the limit of spatial resolution, during imaging when mapping time versus resolution tradeoffs are made, or when the sample is dose sensitive.

The arbitrary nature of collection and processing parameters in EELS and energy-filtered TEM (EFTEM) is troublesome. Error analysis from the most widely used analysis methods often do not yield good precision estimates and rarely are systematic errors accounted for. ${ }^{2}$ Systematic errors can come from processing methods, poor control of analysis variables, and cross-section accuracy. The latter is not treated here, but the other issues can be addressed by analyzing a priori synthesized spectra. Generating ${ }^{3}$ and processing a collection of spectra representing a range of analysis conditions, stable acquisition and processing parameters can be found. Analyzing how synthetically applied noise affects these data sets can give meaningful estimates of non-systematic errors.

Comparing results from the processed data sets to the initial conditions used to generate the data sets gives estimates of systematic errors. We have developed a series of tools to perform these functions, and are working on applying them to the ultimate goal of automated experiment setup and spectral analysis.

As a first step we have sought to address the following questions with these tools, given a priori knowledge of the specimen composition:

1. Can the minimum detectable mass fraction (MMF) and the minimum detectable number of atoms $(\mathrm{MDN})$ be predicted?

2. What experimental conditions should be used to optimize collection of the EELS signal?

3. How should the EELS quantification parameters be chosen to ensure accurate and precise results?

4. As specimen composition, dose (and thus resolution), collection angle, specimen thickness, etc vary, how do systematic and non-systematic errors change?

The answers to the above questions depend on being able to simulate energy loss spectra reliably and accurately and on being able to apply the same methods to quantify the simulated spectra as the experimental spectra. The first version of tool titled "EELSAdvisor" was created for this purpose and includes the capability to optimize most processing and acquisition parameters for EFTEM and EELS experiments utilizing power-law background subtraction. EELSAdvisor is capable of 
addressing questions $1 \& 2$ effectively and we will present experimental verification of results from a variety of conditions (main limitations found come from limitations in detail of simulated spectra). Questions $3 \& 4$ are less effectively addressed because they require significant manual manipulation of the software and too much information comes from this process for casual use. Solving this issue and using it to further automate the analysis process is the focus of current development.

\section{References}

1. R.D. Leapman, J.A. Hunt, Microsc. Microanal. Microstruct, 2 (1991) p231.

2. J.A.Hunt, Microscopy and Microanalysis. 7 Suppl 2: Proc. (2001) p1142.

3. N.K. Menon, O.L. Krivanek, Microscopy and Microanalysis 8 (2002) p203.
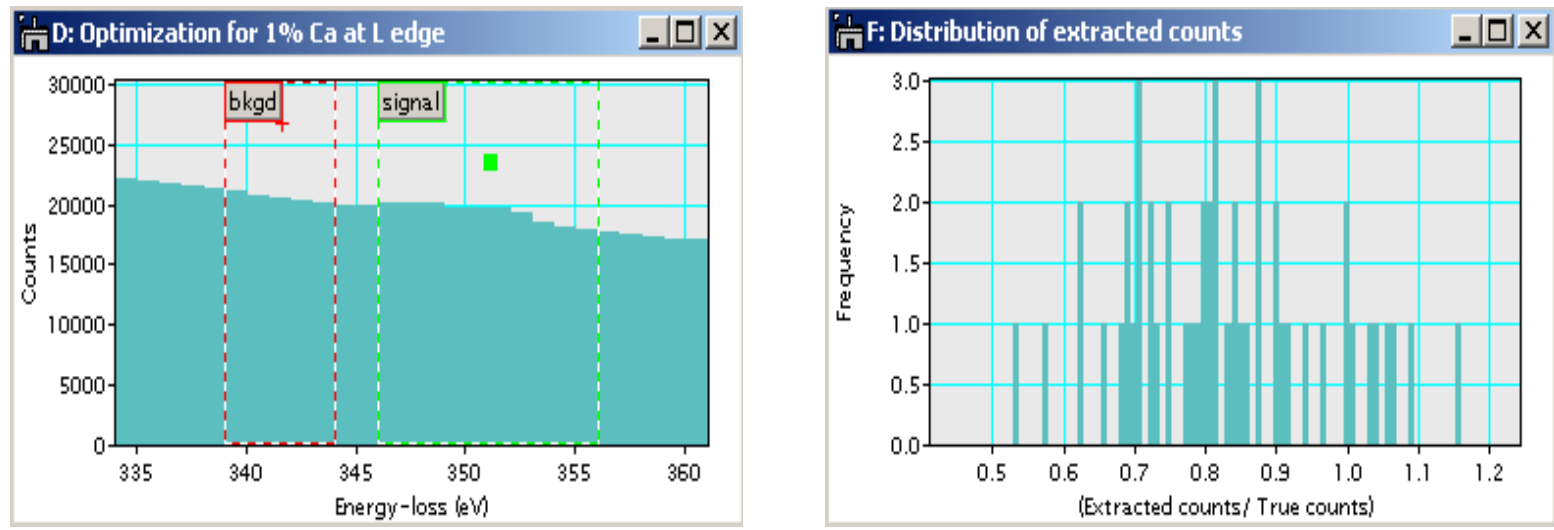

Figure 1. Results from standard EELSAdvisor tool after optimizing processing parameters for detecting $1 \%$ of Ca. (Left) Optimized processing windows; (Right) Distribution of results of many analyses gives estimate of systematic and non-systematic errors - these are used to drive the optimization process.

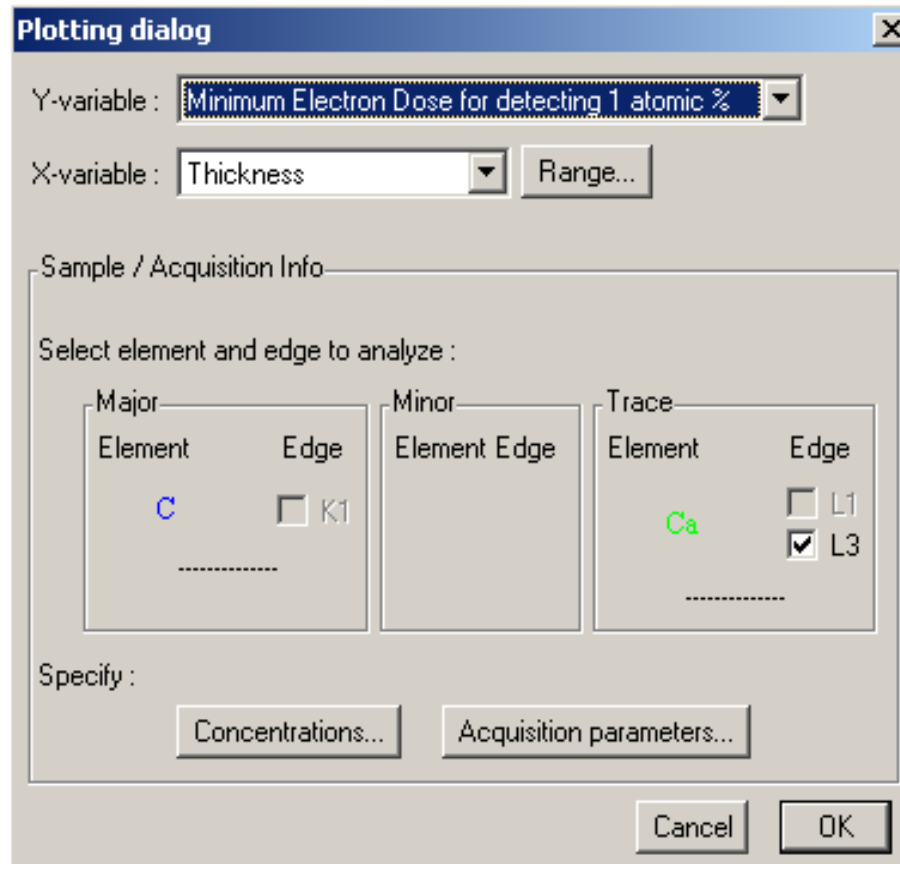

A E: Minimum dose ys thickness for 1 per cent Ca in $[-|\square| x \mid$

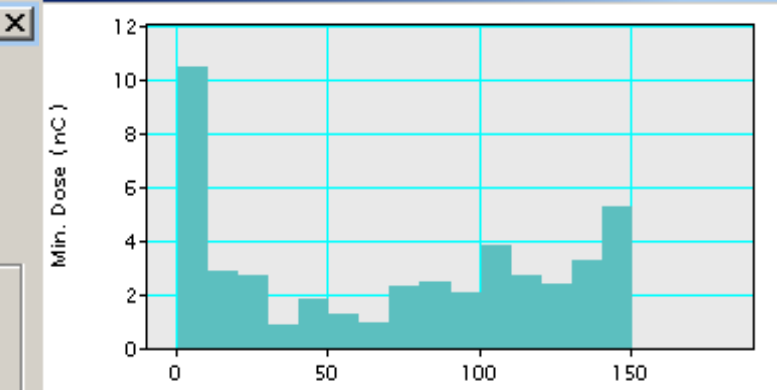

7. G: Minimum dose ys thickness for 10 per cent Ca in... $-|\square| x$

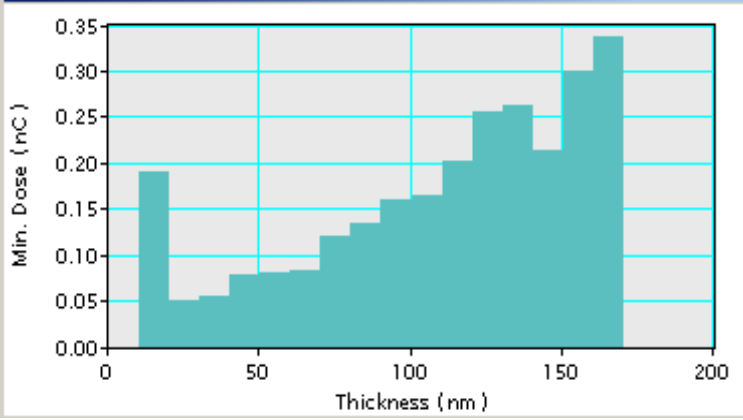

Figure 2. Determining stable acquisition and analysis conditions requires understanding sensitivity to parameters such as thickness. The prototype tool shown is a manual approach to this problem. 